\title{
A Human-Mouse Hybrid HLA-A2-IG Dimer Inhibits the Proliferation of CD4+T Cells In Vitro
}

\author{
Victor Tunje Jeza ${ }^{1}{ }^{2 *}$, Jun Chen ${ }^{2}$, Xiongwen $\mathrm{Wu}^{2}$ \\ 1 Department of Medical Sciences, Technical University of Mombasa, P.O. Box 90420-80100, \\ Mombasa, Kenya; ${ }^{2 T}$ ongji Medical College, Huazhong University of Science and Technology, \\ Wuhan, China \\ *Corresponding author: vjeza@tum.ac.ke
}

\begin{abstract}
C

urrently, general immunosuppressive drugs are used to maintain tolerance to allografts. However, these drugs have a major drawback of rendering the patient susceptible to infections and other side effects like malignancy and drug related toxicities with an overall rejection of the organ at some point. Previous studies have shown that MHC-Ig dimers may suppress alloresponsive T cells in a donor specific manner in vitro. This work aimed to answer the question as to whether these dimers will surmount rejection through the direct mechanism of allorecognition by suppressing alloreactive $C D 8^{+} \mathrm{T}$ cells. To do this, we first identified two mice models with a single mismatch at the MHC loci. We found and procured white albino NOD mice which happened to be transgenic for HLA-A2 and HLA-A24 molecules. We then constructed a human-mouse hybrid HLA-A2-Ig dimer by overlap-PCR to join parts of two different already cloned plasmids to form the full length HLA-A2ß2a1a2murinea3 insert which was then cloned to pcDNA3.1 to form pcDNA3.1HLA$\mathrm{A} 2 \beta 2 \mathrm{a} 1 \mathrm{a} 2 \mathrm{murine} 3 \mathrm{3}$. The IgG2bFc region was added by restriction digestion and ligation to form the plasmid pcDNA3.1HLA-A2 $32 a 1 a 2 m u r i n e a 3 I g G 2 b F c$. Sequencing was done and confirmed that the construction and cloning were successful. The plasmid pcDNA3.1HLA-A2 $22 a 1 a 2 m u r i n e a 3 I g G 2 b F c$ was then transfected by electroporation to J558L cells. Screening was done using G418 for 4 weeks in cell culture. We purified the dimer by affinity chromatography and then used ELISA to confirm expression of the dimer. The purified dimer was then used in 1-way MLC experiments where responder cells were mice cells expressing HLA-A24 molecules while stimulator cells were mice cells expressing HLA-A2 molecules. Cell samples were gated on anti-mouse CD3-PE/CY7, anti-mouse CD4-PE, and anti-mouse CD8-APC/CY7. Cell proliferation was analysed using CFSE. Our results showed that the proliferation of $\mathrm{CD} 4^{+} \mathrm{T}$ cells was inhibited in the presence of the dimer. This work is crucial for subsequent studies aiming to search for induction of donor specific tolerance.
\end{abstract}

Key Words: HLA-A2-Ig, Dimer, HLA-A24, proliferation, CD4+ T cells

\section{Introduction}

Previous studies have shown that suppression of $\mathrm{T}$ cells is peptide-MHC complexes specific and can either be a syngeneic or allogeneic response in an indistinguishable manner (Chen et al., 2009; Heath, et al., 1991; Kourilsky \& Claverie, 1989; X. F. Weng et al., 2007; X. Weng et al., 2007, 2009; Zhong et al., 2009). Further, other studies have shown that the vast majority of alloreactive $\mathrm{T}$ cells react to alloantigens presented by the direct pathway of allorecognition (Benichou et al., 1999; Benichou, 1999; Liu et al., 1993; Marino et al., 2016). This is supported by the realization that the frequency of cells engaged in the indirect pathway of allorecognition is approximately 10 -fold lower than that of cells engaged in direct recognition. As a result, the direct pathway accounts for $>90 \%$ of acute skin graft rejection in a variety of mice models (Benichou et al., 1999; Benichou, 1999; Benichou \& Fedoseyeva, 1996; Marino et al., 2016). In fact, the direct pathway has classically been considered the major driving force in alloresponses (Womer, et al., 2000). There is also some evidence that allorecognition through the direct pathway may actually inhibit allograft rejection at least later in the course of rejection, through inhibition of direct alloresponses (Frasca et al., 1998; Gould \& Auchincloss, 1999; Lee et al., 1997).

We postulated that suppression of donor MHC specific alloresponsive $\mathrm{T}$ cells could abrogate direct 
allograft rejection in vivo. In this regard, we first set out to determine whether donor derived MHC-Ig dimers could suppress donor specific alloreactive $\mathrm{T}$ cells in vitro. Employing a donor specific blockage of the direct response would set a different trend from the current means of using general immunosuppressive agents by allowing nonalloreactive $\mathrm{T}$ cells to survive and be available for other protective roles thereby tremendously reducing the disadvantages of non-specific immunosuppression in allograft transplantation. In this regard, we proposed a novel mechanism for suppressing antigen specific alloreactive $\mathrm{T}$ cells. Our model consisted of transgenic mice; the donor mice expressing the HLA-A2 molecules and recipient mice expressing the HLA-A24 molecules (Fig. 1). To block allograft rejection in a donor antigen specific fashion in our proposed model, we needed a human-mouse hybrid HLA-A2 dimer that is loaded with similar antigens to those of the donor. We therefore embarked on constructing, cloning, and expression of the dimer as initial preparatory steps. To have similar miscellaneous antigens to those of the donor attached to the dimer, the dimer was expressed in J588L cells. This was followed by testing of the ability of the dimer to suppress alloreactive $\mathrm{CD} 8{ }^{+} \mathrm{T}$ cells in vitro using mixed lymphocytes culture (MLC) experiments. Our results show that $\mathrm{CD} 4^{+} \mathrm{T}$ cell proliferation was inhibited by HLA-A2-Ig dimers.

\section{Materials and Methods \\ Construction, Sequencing, Cloning, and Expression of the HLA-A2-Ig Dimer}

The HLA-A2-Ig dimer was constructed from 2 already constructed different plasmids (one of the plasmids contained the HLA-A2- $\beta 2$ a1a2 coding region and the other one contained the mouse $\mathrm{H} 2 \mathrm{D}^{\mathrm{b}} \mathrm{a} 3$ coding region) by amplifying the regions of interest using PCR and joining them to make the HLA-A2- $\beta 2 \alpha 1 \alpha 2 a 3$ insert by overlap-PCR as described previously (G. Pont-Kingdon, 1994; Genevieve Pont-Kingdon, 2003). This was then ligated to the pcDNA3.1 to form the plasmid pcDNA-HLA-A2- $\beta 2 a 1 a 2 H 2 D^{b} a 3$. The IgG2bFc region was also amplified by PCR from an already existing plasmid and then joined to the pcDNA3.1HLA-A2- $32 a 1 a 2 a 3$ plasmid by restriction digestion and ligation to form the pcDNA3.1-HLAA2- $\beta 2 \alpha 1 a 2 a 3 \operatorname{IgG} 2 b F c$ plasmid. The constructed dimer was validated by sequencing (Invitrogen,
Shanghai - China). It was then transfected into J558L cells by electroporation followed by selecting for stable transfected clones for a month using G418 antibiotic. Thereafter, expression of the dimer was done in J558L cells cultured in Rosewell Park Memorial Institute (RPMI) 1640 (Gibco - USA) supplemented with 10\% FBS (Gibco - USA).

\section{ELISA}

ELISA was done to determine the expression of the HLA-A2 dimer after electroporation of J558L cells and selecting for stable transfected clones for a month using G418 antibiotic. Sandwich ELISA was performed using following antibodies in accordance with the manufacturer's instructions: Goat anti-mouse IgG (Biolegend - USA) capture antibody, Rabbit anti-human $\beta 2$-microglobulin (Biolegend - USA) detection antibody, and goat anti-rabbit IgG (Biolegend - USA) secondary antibody.

\section{Processing of the Dimer}

The dimer was harvested from supernatant of confluent grown J558L transfected cells. The supernatant was concentrated using Polyethylene Glycol (PEG) 20000 (BioSharp - USA). It was then dialyzed in PBS overnight at $4^{\circ} \mathrm{C}$ followed by sterilization by sieving through a $2 \mu \mathrm{m}$ sieve and stored at $-20^{\circ} \mathrm{C}$ until use.

\section{Mice}

Strain 1 was NOD.129p2 (B6)-B2m<tmlUnc $>$ Tg (HLAA/H2-D/B2m) 1Dvsj - These mice are transgenic for HLA-A/H2-D/B2M gene and as a result they express the HLA-A2.1 molecules. They are commonly referred to as NOD.HHD. These mice do not express murine MHC class I and therefore all the $\mathrm{CD}^{+} \mathrm{T}$ cells are HLA-A2.1 restricted (Pascolo et al., 1997; Serreze, Marron, \& DiLorenzo, 2007; Takaki et al., 2006). Compared to the standard NOD mice expressing murine MHC class I molecules, these mice have a $90 \%$ reduction in CD8 ${ }^{+}$T cells (Firat, 2002; Serreze et al., 2007; Takaki et al., 2006). Strain 2 was NOD.129p2 (B6)B2m $<$ tmlUnc $>$ Tg (HLA-A24/H2-D/B2m) 3Dvs/jThese mice are transgenic for HLA-A24/H2$\mathrm{D} / \mathrm{B} 2 \mathrm{M}$ gene and homozygous for the $\mathrm{B} 2 \mathrm{~m}^{\mathrm{tm} 1 \mathrm{Unc}}$ mutation. They are commonly referred to as NOD.B2mnull. A24/HHD. Both strains were obtained from Jackson labs in the US and 
propagated at the Tongji Medical College animal house.

\section{One-Way Mixed Lymphocytes Culture (MLC)}

Lymphocytes from the spleens of HLA-A24 mice were processed and stained with CFSE then used as responder cells while those from the spleens of HLA-A2 mice were processed and used as stimulator cells. The co-culture was done in RPMI 1640 (Gibco - USA) supplemented with 10\% FBS (Gibco - USA) at a responder to stimulator ratio of 10:1 with responder cells being $2.5 \times 10^{6}$ cells per well in 24 well plates. The wells were then treated with the dimer and non-transfected J588L supernatant in sets. Sets of non-treated wells were used as negative controls.

\section{Flow Cytometry}

After co-culture for 6 days, samples were stained using PE/CY7 conjugated anti-mouse CD3 (E Bioscience - USA), PE conjugated anti-mouse CD4 (E Bioscience - USA), and APC/CY7 conjugated anti-mouse CD8 (Biolegend - USA) antibodies. Flow cytometry was performed for analysis. Statistical analysis was done using the student's $t$ test. A p value of 0.05 or less was considered significant.

\section{Results \\ HLA-A2-Ig Dimer Construction, Cloning, And Expression}

The dimer was constructed by recombinant DNA technology followed by overlap-PCR and cloned into pcDNA3.1 plasmid. Results were analysed using gel electrophoresis (Fig. 2) and DNA sequencing (Invitrogen, Shanghai, China) (Table 1). The resultant clone was then transfected to J588L cells by electroporation and the expressed dimer analysed by ELISA. Fig. 3 shows that there was almost nothing detected for the negative control (next to last bar on the far right designated J588L). This was the same for phosphate buffered saline (PBS), cell culture media (RPMI 1640), and blank controls. All the samples with transfected plasmid clone (here designated S1 to S5) show high levels of the dimer proteins (Fig. 3). Taken together, these results show that our HLA-A2-Ig dimer was successfully constructed, cloned, and expressed.

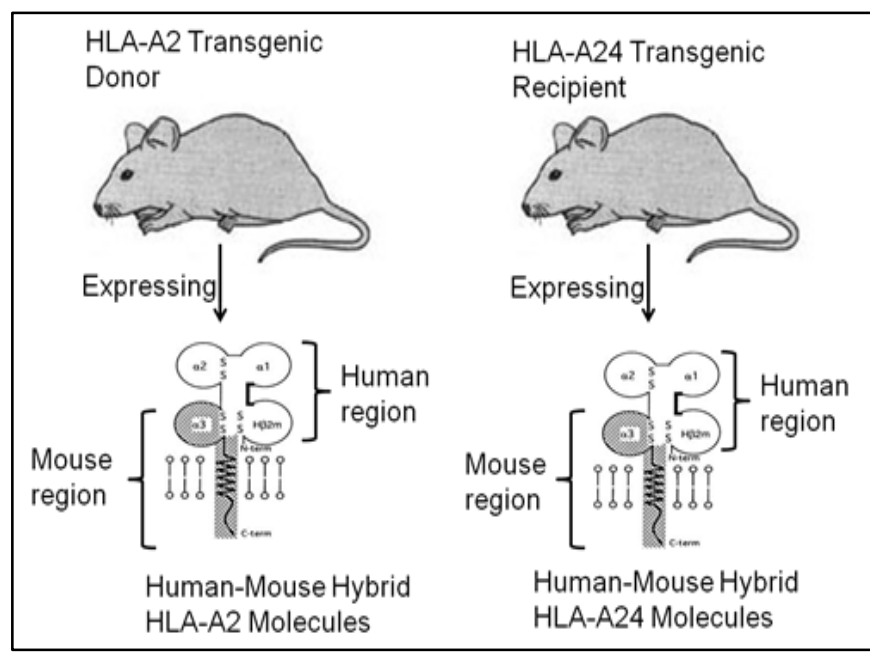

Figure 1. Single locus mismatched allo-transplant model (Jackson lab, NH, USA). A human-mouse hybrid HLAA2 dimer was constructed in order to block allograft rejection in a donor antigen specific fashion in this model

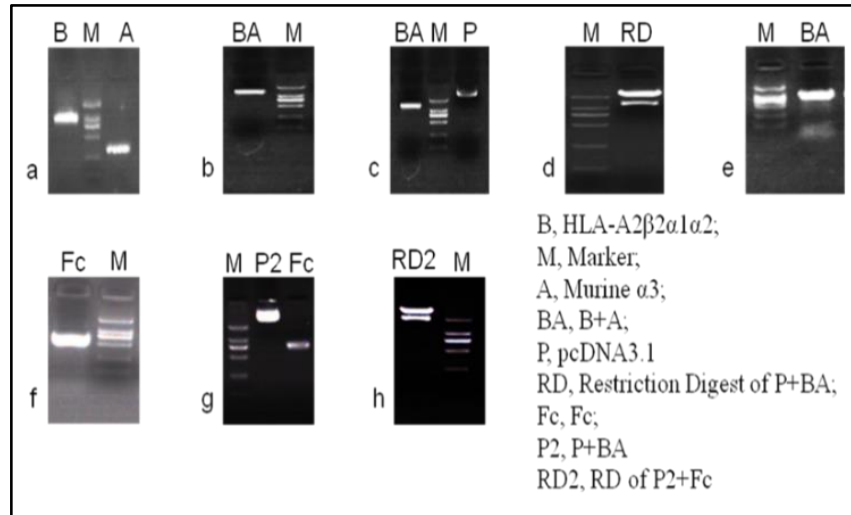

Figure 2. Gel electrophoreses of PCR products during the construction of the pcDNA3.1-HLA-

A2ß2a1a2murinea3-Fc dimer

(a) Amplification of the HLA-A2 $\beta 2 \alpha 1 \alpha 2$ region and the murinea 3 region gave a $1 \mathrm{~kb}$ and $0.25 \mathrm{~kb}$ fragment respectively. (b) Joining of the HLAA2 $32 a 1 a 2$ fragment and the murinea 3 fragment by overlap PCR gave a 1.25 fragment which is the HLA-A2 32 a1a2murinea3 region. (c) Digestion of the HLA-A2 $32 \alpha 1 \alpha 2 m u r i n e a 3$ and the pcDNA3.1 plasmid with HindIII and EcoRV to create cohesive ends before forming the pcDNA3.1-HLAA2ß2a1a2murinea3 by ligation. (d) Checking by digesting with HindIII and EcoRV restriction enzymes showed that the ligation and transformation to DH5a competent cells was successful. (e) Checking by PCR showed the HLA- 
A2ß2a1a2murinea3 was correct at $1.25 \mathrm{~kb}$. (f) Amplification of the Fc fragment using FcRRR and FcXbaIRR enzymes which contained the EcoRV and $\mathrm{XbaI}$ restriction enzyme sites (see Table 1 ) gave a $0.75 \mathrm{~kb}$ fragment. (g) Restriction digesting with EcoRV and XbaI enzymes to create cohesive ends. This was followed by ligation to form the pcDNA3.1-HLA-A2ß2a1a2murinea3-Fc plasmid which was then transformed to DH5a competent cells. (h) Digestion of the pcDNA3.1-HLAA2ß2a1a2murinea3-Fc plasmid with HindIII and XbaI enzymes gave a $2 \mathrm{~kb}$ fragment which represented the HLA-A2ß2a1a2murinea3-Fc region. This confirmed that the ligation and transformation procedures were successful.

Table 1. The sequence of the constructed HLA-A2$\beta 2 a 1 a 2-m o u s e a 3 \operatorname{IgG} 2 b F c$ region. Sequencing was done by the Invitrogen Company in Shanghai, China. The correct sequence confirmed that all procedures were successfully carried out

ATGTCTCGCT CCGTGGCCTT AGCTGTGCTC GCGCTACTCT CTCTTTCTGG CCTGGAGGCT ATCCAGCGT CTCCAAAGAT TCAGGTTTAC TCACGTCATC CAGCAGAGAA TGGAAAGTCA AATTTCCTGA ATTGCTATG GTCTGGGTTT CATCCATCCG ACATTGAAGT TGACTTACTG AAGAATGGAG AGAGAATTGA AAAAGTGGA CATTCAGACT TGTCTTTCAG CAAGGACTGG TCTTTCTATC TCTTGTACTA CACTGAATTC ACCCCCACT AAAAAGATGA GTATGCCTGC CGTGTGAACC ATGTGACTTT GTCACAGCCC AAGATAGTTA AGTGGGATC AGACATGTCA GGTGGTGGCG GATCCGGCGG AGGTGGCTCT GGCGGTGGCG GATCGGGCTC TCACTCCAT AGGTATTTCT TCACATCCGT GTCCCGGCCC GGCCGCGGGG AGCCCCGCTT CATCGCAGTG GGCTACGTG ACGACACGCA GTTCGTGCGG TTCGACAGCG ACGCCGCGAG CCAGAGGATG GAGCCGCGGG CGCCGTGG AGAGCAGGAG GGTCCGGAGT ATTGGGACGG GGAGACACGG AAAGTGAAGG CCCACTCAC GACTCACCGA GTGGACCTGG GGACCCTGCG CGGCTACTAC AACCAGAGCG AGGCCGGTTC TCACACCGT CAGAGGATGT ATGGCTGCGA CGTGGGGTCG GACTGGCGCT TCCTCCGCGG GTACCACCAG TACGCCTAC ACGGCAAGGA TTACATCGCC CTGAAAGAGG ACCTGCGCTC TTGGACCGCG GCGGACATGG CAGCTCAGA CACCAAGCAC AAGTGGGAGG CGGCCCATGT GGCGGAGCAG TTGAGAGCCT ACCTGGAGGG CACGTGCGT CACCAAGCAC AAGTGGGAGG CGGCCCATGT GGCGGAGCAG TTGAGAGCCT ACCTGGAGGG CACGTGCGT TGACCCATCA CCCCAGATCT AAAGGTGAAG TCACCCTGAG GTGCTGGGCC CTGGGCTTCT ACCCTGCTG CATCACCCTG ACCTGGCAGT TGAATGGGGA GGAGCTGACC CAGGACATGG AGCTTGTGGA GACCAGGCC GCAGGGGATG GAACCTTCCA GAAGTGGGCA TCTGTGGTGG TGCCTCTTGG GAAGGAGCAG AATTACAC $A$ GCCGTGTGTA CCATGAGGGG CTGCCTGAGC CCCTCACCCT GAGATGGGAG CCTCCTCCGT CCACTGACI TTACATGGTG GATATCGAGC CCAGCGGGCC CATTTCAACA ATCAACCCCT GTCCTCCATG CAAGGAGTG CACAAATGCC CAGCTCCTAA CCTCGAGGGT GGACCATCCG TCTTCATCTT CCCTCCAAAT ATCAAGGAT TACTCATGAT CTCCCTGACA CCCAAGGTCA CGTGTGTGGT GGTGGATGTG AGCGAGGATG ACCCAGACC CCAGATCAGC TGGTTTGTGA ACAACGTGGA AGTACACACA GCTCAGACAC AAACCCATAG AGAGGATTA AACAGTACTA TCCGGGTGGT CAGCACCCTC CCCATCCAGC ACCAGGACTG GATGAGTGGC AAGGAGTTC AATGCAAGGT CAACAACAAA GACCTCCCAT CACCCATCGA GAGAACCATC TCAAAAATTA AAGGGCTAG CAGAGCTCCA CAAGTATACA TCTTGCCGCC ACCAGCAGAG CAGTTGTCCA GGAAAGATGT CAGTCTCAC TGCCTGGTCG TGGGCTTCAA CCCTGGAGAC ATCAGTGTGG AGTGGACCAG CAATGGGCAT ACAGAGGAG ACTACAAGGA CACCGCACCA GTCCTGGACT CTGACGGTTC TTACTTCATA TATAGCAAGC TCAATATGA AACAAGCAAG TGGGAGAAAA CAGATTCCTT CTCATGCAAC GTGAGACACG AGGGTCTGAA AAATTACTA CTGAAGAAGA CCATCTCCCG GTCTCCGGGT AAATAA

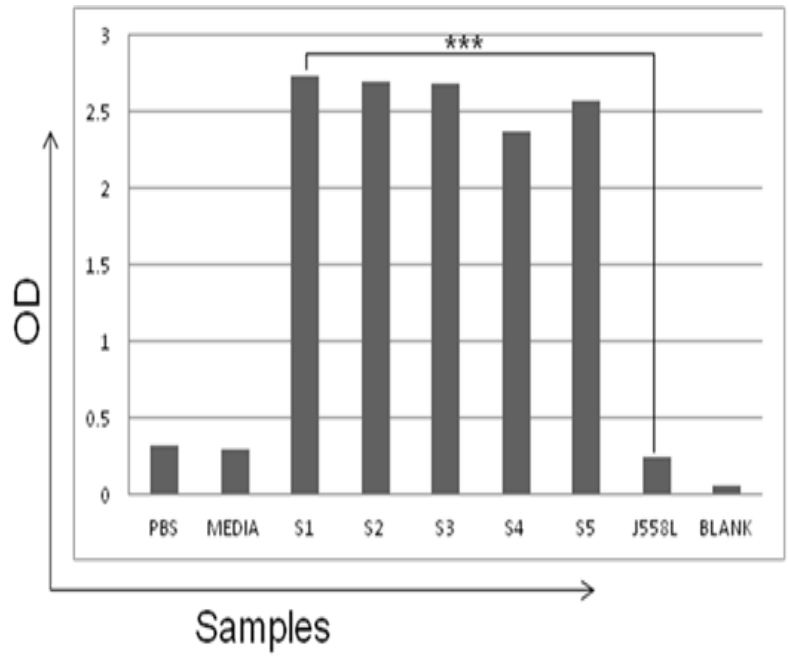

Figure 3. ELISA results of the dimer expression by J558L cells. S1 to S5 are supernatant samples from different clones of the transfected J558L cells while J558L represents the supernatant sample from non-transfected J558L cells.

\section{Inhibition of CD4+ T Cell Proliferation}

Lymphocytes from the spleens of HLA-A24 mice were processed and stained with CFSE then used as responder cells while those from the spleens of HLA-A2 mice were processed and used as stimulator cells. After co-culture for 6 days, samples were stained using anti-mouse CD3 (PE/CY7 conjugated), anti-mouse CD4 (PE conjugated), and anti-mouse CD8 (APC/CY7 conjugated) antibodies. Only cells containing CD3 and CD4 molecules were observed while those containing CD3 and CD8 were not detectable. Proliferation magnitude was determined using the proliferation index (PI) (Saverino et al., 2002). As shown in figure 4, the PI for dimer treated samples was statistically significantly lower than control samples with a $\mathrm{p}$ value of 0.00158 (Fig. 4). Taken together, these results show that the dimer inhibited the proliferation of CD4+ T cells. 


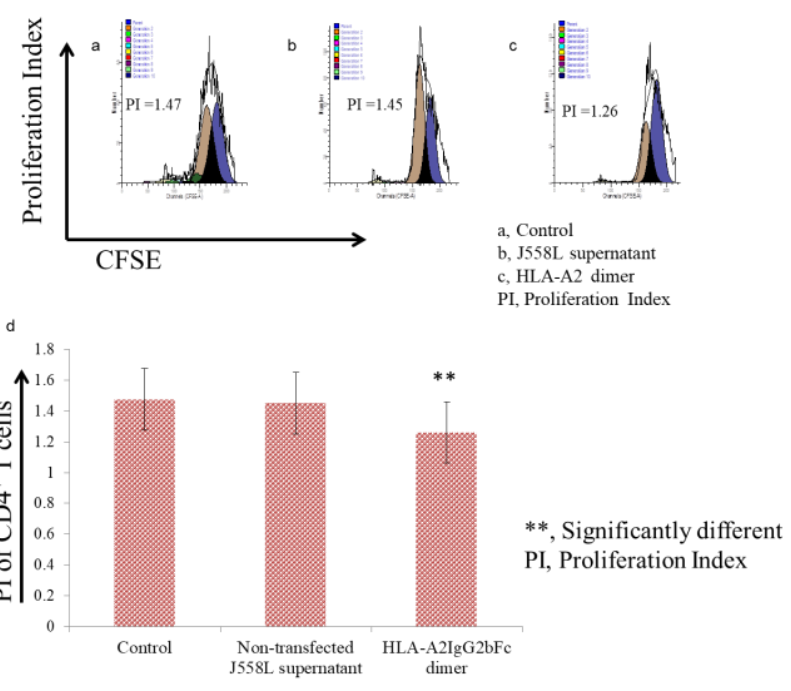

Figure 4. Proliferation of $\mathrm{CD} 4^{+} \mathrm{T}$ cells The proliferation of $\mathrm{CD}^{+} \mathrm{T}$ cells was inhibited in dimer treated samples $(\mathrm{p}=0.00158)$ compared to control samples. At the same time, the proliferation of CD4+ T cells was not statistically significant for non-transfected J588L cell supernatant ( $\mathrm{p}=0.09585)$ compared to control samples. (a) Control (media only). (b) non-transfected J558L supernatant. (c) HLA-A2IgG2bFc dimer. (d) graphical representation of the proliferation indices.

\section{Discussion}

Currently, organ transplantation offers hope to end stage failed organs and tissues. Although the art of surgical transfer of the organs from one organism to another has been mastered, the immune system poses the biggest risk to the resultant transplant. To counter the effects of the immune system to the transplanted organs, general immunosuppressive drugs are normally used. However, this poses the threat of lowering the overall ability of the immune system to protect the individual and therefore results in susceptibility to infections and noninfectious diseases like cancer. It is therefore imperative to come up with strategies that can either reduce our reliance on immunosuppressive drugs or remove the need to use them altogether.

Previous studies have shown that HLA-A2-Ig dimers can inhibit alloreactive $\mathrm{T}$ cell responses in vitro (X. Weng et al., 2007; Zhong et al., 2009). In this regard, we developed a strategy for suppressing alloreactive $T$ cells in vitro that would mimic the reality of the situation in a transplantation environment. We used transgenic mice expressing HLA-A2 and HLA-A24 molecules on their cell surface (Fig. 1). These were therefore single locus mismatched which closely mimic transplantation environments with the difference of having just one mismatch instead of several more even in the cases of closely matching the organ and the recipient in the real situation. The single locus polymorphism here would still allow for activation of alloreactive $\mathrm{T}$ cells.

We constructed a HLA-A2-Ig dimer which would then interact with the responder cells. To ensure that we had the correct dimer molecule, a series of methods for construction and validation were used (Fig. 2, Table 1, and Fig. 3) which all confirmed it was right. This was then applied in the single mismatch transplantation model where the responder cells represented the recipient while the stimulators cells represented the donor. Our results showed that the HLA-A2 dimer significantly reduced the proliferation of the responder $\mathrm{T}$ cells containing CD4 molecules (Fig. 4). We expected to see a reduction in $\mathrm{CD}^{+} \mathrm{T}$ cells but this did not occur. One possible explanation for the phenomenon we observed could be through trogocytosis. It has been shown that cell membrane molecules could be transferred to other cells by a process of trogocytosis (Chung et al., 2014; Dopfer, Minguet, \& Schamel, 2011; Gilmartin \& Petri Jr, 2017; Matlung et al., 2018; Miyake et al., 2017; Perry \& Ravichandran, 2017). Other cells could have acquired TCRs or CD4 molecules by the process of trogocytosis which were (other cells) then able to interact with the HLA-A2-Ig dimer molecules leading to the observation made. It has been shown that the direct pathway accounts for $>90 \%$ of acute skin graft rejection in a variety of mice models (G Benichou et al., 1999; Gilles Benichou, 1999; Gilles Benichou \& Fedoseyeva, 1996). Cells involved in this model are $\mathrm{CD}^{+} \mathrm{T}$ cells. The indirect mechanism of antigen presentation is done mainly through the $\mathrm{CD}^{+} \mathrm{T}$ cells. Suppressing both $\mathrm{CD} 4^{+}$ and $\mathrm{CD}^{+} \mathrm{T}$ cells would be more appealing. Therefore, the ability to suppress $\mathrm{CD} 4^{+} \mathrm{T}$ cells is encouraging since these cell types are the biggest players in transplantation rejection through the chronic mechanism made possible by the indirect presentation of antigens to T cells. These results are therefore crucial for future studies that aim to develop strategies for donor specific tolerance induction. However, more studies are needed to elucidate the mechanism by which $\mathrm{CD}^{+}{ }^{+} \mathrm{T}$ cell proliferation was down-regulated. 


\section{Acknowledgement}

This work was supported in part by the Chinese Government Scholarship (CSC) through the Kenya Government, Prof. Wu's Laboratory at Tongji Medical College, and Technical University of Mombasa, Kenya.

\section{References}

Benichou, G., Valujskikh, A. \& Heeger, P.S. (1999). Contributions of direct and indirect $\mathrm{T}$ cell alloreactivity during allograft rejection in mice. Journal of Immunology, 162(1), 352-358. Retrieved from http://www.ncbi.nlm.nih.gov/pubmed/988 6406

Benichou, G. (1999). Direct and Indirect Antigen Recognition: the Pathways to Allograft Immune Rejection. Frontiers in Bioscience, 4, 476-480.

Benichou, G. \& Fedoseyeva, E.V. (1996). The contribution of peptides to $\mathrm{T}$ cell allorecognition and allograft rejection. International Reviews of Immunology, Vol. 13, pp. 231-243. https://doi.org/10.3109/08830189609061750

Chen, X., Yan, Y., Lu, S., Weng, X., Liang, Z., Li, J., ... Wu, X. (2009). Raising allo-restricted cytotoxic $\mathrm{T}$ lymphocytes by co-culture of murine splenocytes with autologous macrophage bearing the peptide/allo-major histococompatibility complex. Human Immunology, Vol. 70, pp. 79-84. https://doi.org/10.1016/j.humimm.2008.11. 005

Chung, B., Stuge, T.B., Murad, J. P., Beilhack, G., Andersen, E., Armstrong, B. D., ... Lee, P. P. (2014). Antigen-specific inhibition of highavidity $\mathrm{T}$ cell target lysis by low-avidity $\mathrm{T}$ cells via trogocytosis. Cell Reports, 8(3), 871882.

https://doi.org/10.1016/j.celrep.2014.06.052

Dopfer, E.P., Minguet, S., \& Schamel, W.W.A. (2011). A New Vampire Saga: The Molecular Mechanism of T Cell Trogocytosis. Immunity, 35(2), 151-153. https://doi.org/10.1016/j.immuni.2011.08.0 04

Firat, H. (2002). Comparative analysis of the CD8+ $\mathrm{T}$ cell repertoires of $\mathrm{H}-2$ class $\mathrm{I}$ wildtype/HLA-A2.1 and H-2 class I knockout/HLA-A2.1 transgenic mice.
International Immunology, 14(8), 925-934. https://doi.org/10.1093/intimm/dxf056

Frasca, L., Amendola, A., Hornick, P., Brookes, P., Aichinger, G., Marelli-Berg, F., ... Lombardi, G. (1998). Role of donor and recipient antigenpresenting cells in priming and maintaining $t$ cells with indirect allospecificity. Transplantation, 66(9), 1238-1243. https://doi.org/10.1097/00007890199811150-00020

Gilmartin, A.A. \& Petri Jr, W.A. (2017). Exploring the mechanism of amebic trogocytosis: the role of amebic lysosomes. Microbial Cell, 8(4), 2017-2019. https://doi.org/10.1128/mBio.01187-17

Gould, D.S. \& Auchincloss, H. (1999). Direct and indirect recognition: The role of $\mathrm{MHC}$ antigens in graft rejection. Immunology Today, Vol. 20, pp. 77-82. https://doi.org/10.1016/S01675699(98)01394-2

Heath, W.R., Kane, K.P., Mescher, M.F. \& Sherman, L. A. (1991). Alloreactive T cells discriminate among a diverse set of endogenous peptides. Proceedings of the National Academy of Sciences of the United States of America, 88(12), 51015105. https://doi.org/10.1073/pnas.88.12.5101

Kourilsky, P., \& Claverie, J.M. (1989). MHCAntigen Interaction: What Does the $\mathrm{T}$ Cell Receptor See? Advances in Immunology, Vol. 45, pp. 107-122. https://doi.org/10.1016/S00652776(08)60693-8

Lee, R.S., Grusby, M.J., Laufer, T.M., Colvin, R., Glimcher, L.H., \& Auchincloss, H. (1997). CD8+ effector cells responding to residual class I antigens, with help from CD4+ cells stimulated indirectly, cause rejection of "major histocompatibility complex-deficient" skin grafts. Transplantation, Vol. 63, pp. 11231133. https://doi.org/10.1097/00007890199704270-00012

Liu, Z., Sun, Y. K., Xi, Y. P., Maffei, A., Reed, E., Harris, P. \& Suciu-Foca, N. (1993). Contribution of direct and indirect recognition pathways to $\mathrm{T}$ cell alloreactivity. Journal of Experimental Medicine, 177(6), 1643 1650.

https:// doi.org/10.1084/jem.177.6.1643 
Marino, J., Paster, J. \& Benichou, G. (2016). Allorecognition by $\mathrm{T}$ lymphocytes and allograft rejection. Frontiers in Immunology, 7(DEC), $1-9$. https://doi.org/10.3389/fimmu.2016.00582

Matlung, H.L., Babes, L., Zhao, X.W., van Houdt, M., Treffers, L.W., van Rees, D.J., ... van den Berg, T. K. (2018). Neutrophils Kill AntibodyOpsonized Cancer Cells by Trogoptosis. Cell Reports, 23(13), 3946-3959.e6. https://doi.org/10.1016/j.celrep.2018.05.082

Miyake, K., Shiozawa, N., Nagao, T., Yoshikawa, S., Yamanishi, Y. \& Karasuyama, H. (2017). Trogocytosis of peptide-MHC class II complexes from dendritic cells confers antigen-presenting ability on basophils. Proceedings of the National Academy of Sciences of the United States of America, 114(5), 11111116.

https://doi.org/10.1073/pnas.1615973114

Pascolo, S., Bervas, N., Ure, J.M., Smith, A.G., Lemonnier, F. A., \& Pérarnau, B. (1997). HLAA2.1-restricted Education and Cytolytic Activity of CD8. Journal of Experimental Medicine, 185(12), 2043-2205.

Perry, J.S.A. \& Ravichandran, K.S. (2017). Embryonic Trogocytosis: Neighborly Nibbling during Development. Current Biology, 27(2), R68-R70. https://doi.org/10.1016/j.cub.2016.11.043

Pont-Kingdon, G. (1994). Construction of chimeric molecules by a two-step recombinant PCR method. BioTechniques, Vol. 16, pp. 1010-1011.

Pont-Kingdon, Genevieve. (2003). Creation of chimeric junctions, deletions, and insertions by PCR. Methods in Molecular Biology (Clifton, N.J.), Vol. 226, pp. 511-516. https://doi.org/10.1385/1-59259-384-4:511

Saverino, D., Merlo, A., Bruno, S., Pistoia, V., Grossi, C. E. \& Ciccone, E. (2002). Dual Effect of CD85/Leukocyte Ig-Like Receptor-1/IgLike Transcript 2 and CD152 (CTLA-4) on Cytokine oduction by Antigen-Stimulated Human T Cells. The Journal of Immunology, 168(1), 207-215. https://doi.org/10.4049/jimmunol.168.1.207

Serreze, D.V., Marron, M.P. \& DiLorenzo, T.P. (2007). "Humanized" HLA transgenic NOD mice to identify pancreatic $\beta$ cell autoantigens of potential clinical relevance to type 1 diabetes. Annals of the New York Academy of Sciences, 1103, 103-111. https://doi.org/10.1196/annals.1394.019

Takaki, T., Marron, M.P., Mathews, C.E., Guttmann, S.T., Bottino, R., Trucco, M., ... Serreze, D.V. (2006). HLA-A*0201-Restricted $\mathrm{T}$ Cells from Humanized NOD Mice Recognize Autoantigens of Potential Clinical Relevance to Type 1 Diabetes. The Journal of Immunology, 176(5), 3257-3265. https://doi.org/10.4049/jimmunol.176.5.325 7

Weng, X.F., Liang, Z.H., Lu, X.L., Zhong, M.H., Lu, S.J., Zhang, C.E., ... Gong, F.L. (2007). Peptide-specific, allogeneic $\mathrm{T}$ cell response in vitro induced by a self-peptide binding to HLA-A2. Science in China, Series C: Life Sciences, 50(2), 203-211. https://doi.org/10.1007/s11427-007-0036-y

Weng, X., Zhong, M., Liang, Z., Lu, S., Hao, J., Chen, X., ... Wu, X. (2007). Peptide-dependent inhibition of alloreactive $\mathrm{T}$-cell response by soluble divalent HLA-A2/IgG molecule in vitro. Transplantation, 84(10), 1298-1306. https://doi.org/10.1097/01.tp.0000288231.97 $880 . b d$

Weng, X., Lu, S., Zhong, M., Liang, Z., Shen, G., Chen, J., \& Wu, X. (2009). Allo-restricted CTLs generated by coculturing of PBLs and autologous monocytes loaded with allogeneic peptide/HLA/IgG1-Fc fusion protein. Journal of Leukocyte Biology, 85(3), 574-581. https://doi.org/10.1189/jlb.0408242

Womer, K.L., Nadim, M.K. \& Sayegh, M.H. (2000). T-cell recognition of allograft target antigens. Current Opinion in Organ Transplantation, Vol. 5, pp. 23-28. https://doi.org/10.1097/00075200200003000-00005

Zhong, M., Weng, X., Liang, Z., Lu, S., Li, J., Chen, $X ., \ldots W u, X .(2009)$. Dimerization of soluble HLA-G by IgG-Fc fragment augments ILT2mediated inhibition of T-cell alloresponse. Transplantation, 87(1), 8-15. https://doi.org/10.1097/TP.0b013e31818b61 41 